\title{
Erratum to: A variant of the Hough Transform for the combined detection of corners, segments, and polylines
}

Pilar Bachiller-Burgos", Luis J. Manso and Pablo Bustos

\section{Erratum}

Upon publication of the original article [1], it was noticed that in the caption of Fig. 2, there is a typographical error in the expression " $\mathrm{p} \geq \int \mathrm{p} \mathrm{i}$ " that defines the cell positions for which a point ei should vote. The correct expression is " $\mathrm{p}>=\mathrm{pi}$ " This has now been acknowledged and corrected in this erratum. The original article has been corrected.

Received: 11 May 2017 Accepted: 11 May 2017

Published online: 19 May 2017

\section{Reference}

1. P Bachiller-Burgos, LJ Manso, P Bustos, A variant of the Hough Transform for the combined detection of corners, segments, and polylines. EURASIP Journal on Image and Video Processing 2017(1), 32 (2017)

\footnotetext{
* Correspondence: pilarb@unex.es

Department of Computer and Communication Technology, University of Extremadura, Caceres, Spain
} 